\title{
Oxygen Ion Implantation in Strontium Bismuth Tantalate Thin Films
}

\author{
L. Rico*, B. J. Gómez*, M. Stachiotti ${ }^{* \dagger}$, N. Pellegri* ${ }^{* \dagger}$ J. N. Feugeas*, and O. de Sanctis*† \\ *Instituto de Física Rosario (CONICET-Universidad Nacional de Rosario), \\ Bvrd. 27 de Febrero 210 Bis, S2000EZP Rosario, Argentina \\ ${ }^{\dagger}$ Laboratorio de Materiales Cerámicos, Universidad Nacional de Rosario, Pellegrini 250, S2000EZP Rosario, Argentina
}

Received on 8 December, 2005

\begin{abstract}
Strontium Bismuth Tantalate (SBT) ferroelectric thin films have attracted considerable attention for the development of non-volatile ferroelectric random access memories (NV-FRAMs). These films, however, have a critical problem: a high processing temperature $\left(>700^{\circ} \mathrm{C}\right)$ is required for the crystallization of the perovskite phase. The thermal evolution of the SBT films prepared by Chemical Solution Deposition (CSD) techniques showed the formation of an intermediate oxygen-deficient fluorite phase at $\sim 550^{\circ} \mathrm{C}$. The SBT perovskite phase crystallizes at higher temperatures. To favor an earlier perovskite crystallization, SBT thin films were implanted with oxygen ions pulses produced by a Plasma Focus $(1 \mathrm{~kJ})$. The samples were annealed at different temperatures in oxygen atmosphere and characterized by GI-XRD and Atomic Force Microscopy techniques. It was found that oxygen addition into the SBT structure promotes a better crystallization of the perovskite phase.
\end{abstract}

Keywords: Ferroelectric; Thin films; SBT; Oxygen implantation; Plasma focus

\section{INTRODUCTION}

Strontium Bismuth Tantalate (SBT) ferroelectric thin films have attracted considerable attention for the development of non-volatile ferroelectric random access memories (NVFRAMs) due to its relatively low voltage operation, low leakage current, fast switching and good fatigue resistance with $\mathrm{Pt}$ bottom electrodes [1,2]. However, SBT films have some critical problems such as high processing temperatures $\left(>700^{\circ} \mathrm{C}\right)$ to be applied to semiconductor process and a low remanent polarization which is insufficient for the high density integration of FRAMs. In addition, the control of stoichiometry in SBT films is still a tricky subject because of the lost of the bismuth component with diffusion into the Pt bottom electrode or evaporation during heat-treatment. In order to circumvent these shortcomings, many strategies have been employed such as compositional changes and modifications of the synthesis conditions.

Chemical Solution Deposition (CSD) techniques are interesting routes for the evaluation of new materials and novel strategies due to their versatility, simplicity and low temperature material synthesis [3]. Recently, we reported a stable and simple chemical route for the fabrication of SBT powders [4] and thin films [5,6] using non-hydrolyzing precursors of bismuth, strontium and tantalum alkoxides as starting materials. The utilization of acetoin, one of the Hydroxyketones, as chelating agent produced a transformation path amorphous $\rightarrow$ fluorite $\rightarrow$ perovskite with increasing temperature without segregation of transient bismuth or appearance of secondary phases. After the elimination of the last residual organic groups, that occurs approximately at $430^{\circ} \mathrm{C}$, the crystallization of the oxygen-defective fluorite phase starts. The fluorite $\rightarrow$ perovskite transformation takes place at $\sim 600^{\circ} \mathrm{C}$, together with a small mass gain detected from Thermogravimetric Analysis (TGA) measurements [4]. This mass gain was assigned to the incorporation of oxygen atoms into the fluorite structure. Therefore, it was inferred that an oxygen gain destabilizes the intermediate fluorite phase increasing the driving force for the fluorite $\rightarrow$ perovskite transformation.

The aim of this work is to investigate the effects of oxygen incorporation, by ion implanting, on the crystallization behaviour of SBT thin films. The oxygen implantation was performed during the fluorite phase formation.

\section{EXPERIMENTAL}

Tantalum ethoxide $\left(\mathrm{Ta}\left(\mathrm{OCH}_{2} \mathrm{CH}_{3}\right)_{5}\right.$, purity $99.99 \%$, Aldrich) was initially dissolved in methanol under nitrogen atmosphere. Acetoin (3-Hydroxy-2-butanone, $\mathrm{CH}_{3} \mathrm{COCH}(\mathrm{OH}) \mathrm{CH}_{3}, \mathrm{~A} 1,795-1$, Aldrich) was added to the tantalum-methanol solution (the molar ratio of the chemical modifier to tantalum ethoxide was $\mathrm{R}=4$ ). Then, the strontium acetate [ $\mathrm{Sr}\left(\mathrm{CH}_{3} \mathrm{COO}\right)_{2} .1 / 2 \mathrm{H}_{2} \mathrm{O}$, purity $99.9 \%$, Aldrich] was added. Separately, bismuth nitrate $\left[\mathrm{BI}(\mathrm{NiO} 3)_{3} 5 \mathrm{H}_{2} 0\right.$, purity $99.99 \%$, Aldrich) was dissolved in acetic acid. Finally, the bismuth solution was added dropwise to the tantalumstrontium solution with continuous stirring. The metal precursors were mixed to form a Bismuth-rich SBT solution with a target composition of $\mathrm{Sr}_{0.8} \mathrm{Bi}_{2.2} \mathrm{Ta}_{2} \mathrm{O}_{9}$

Platinized silicon wafers $\left(\mathrm{Pt} / \mathrm{Ti} / \mathrm{SiO}_{2} / \mathrm{Si}\right)$ were used as substrates. To obtain the films, the SBT precursor solution was deposited by spin coating (3000 rpm, $30 \mathrm{~s}$ ) inside a clean bench. The deposition of each layer was followed by thermal treatments in air, at $250^{\circ} \mathrm{C}$ for 5 minutes, at $400^{\circ} \mathrm{C}$ for 10 minutes and $520^{\circ} \mathrm{C}$ for 20 minutes. Crack free films of 200 $\mathrm{nm}$ thickness (measured by AFM) were obtained.

The Oxygen implantation was performed with a Dense Plasma Focus of $1 \mathrm{~kJ}$ (see Fig. 1) by mounting the samples in a holder that allows locate them at $160 \mathrm{~mm}$ in front of the anode (inner electrode) (see experimental setup in references $[7,8]$ ). The capacitors bank charge was $15 \mathrm{kV}$, the pre-vacuum pressure was $70 \mathrm{mTorr}$, the filling gas was oxygen and the pressure was 300mTorr. Samples were implanted with different numbers of energetic plasma pulses of $200 \mathrm{~ns}$.

Finally, the oxygen implanted and non-implanted films 
were annealed at increasing temperatures in oxygen atmosphere for 120 minutes.

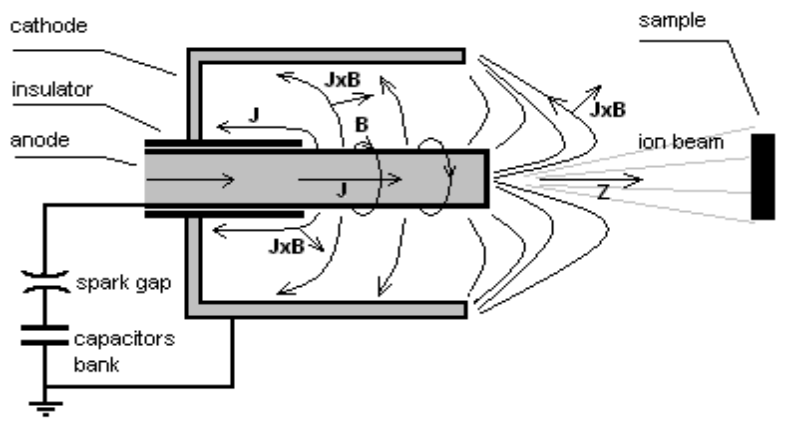

FIG. 1: Plasma Focus devise used for oxygen implantation.
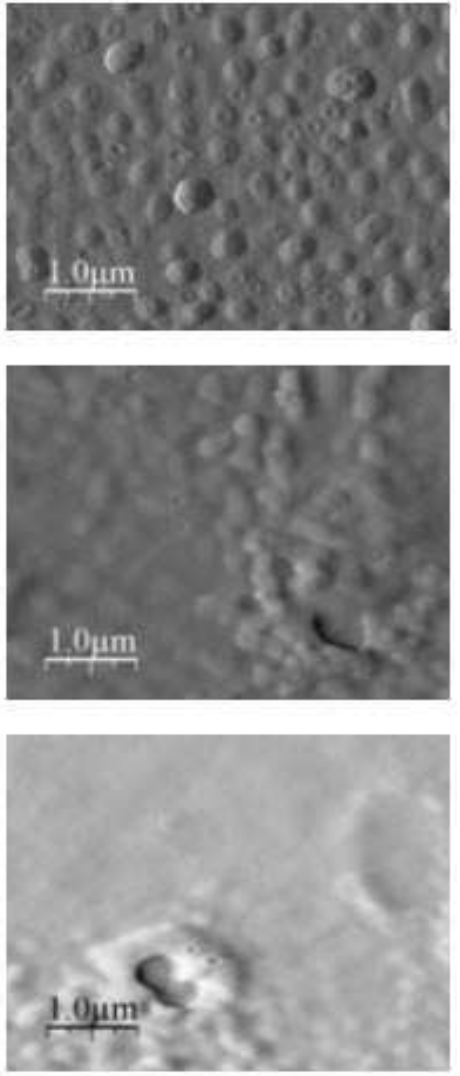

FIG. 2: AFM images of (from top to bottom): a) sample S0 non implanted; b) sample S1 implanted with one pulse of oxygen; c) sample S6 implanted with 6 pulses.

A Chemical analysis of the films was preformed using an EDS Thermo- Noran, SEM LEITZ ARM 1000. Topographic images were observed by AFM using a NanoTec ELECTRONICA equipment working in contact mode configuration, in air at room temperature. A SiN tip supported by a silicon cantilever $\left(0.76 \mathrm{Nm}^{-1}\right.$ spring constant) was used. The XRD data were collected on a Phillips X'pert diffractometer using
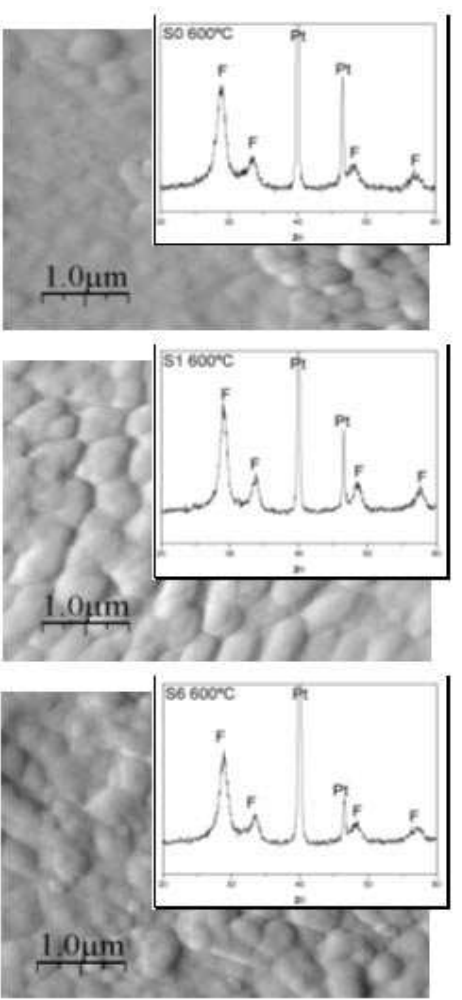

FIG. 3: AFM images and XRD spectrums of samples annealing at $600^{\circ} \mathrm{C}$ (from top to bottom): a)S0; b)S1; c)S6.

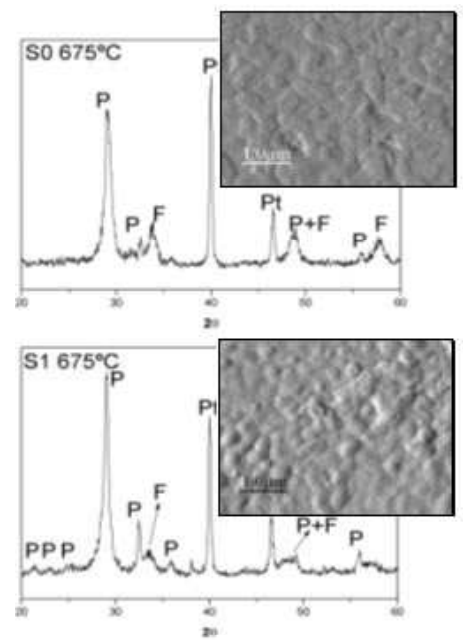

FIG. 4: XRD spectrums and AFM images of samples annealing at $675^{\circ} \mathrm{C}$ (from top to bottom):a) S0; b) S1.

$\mathrm{CuK}_{\alpha}$ radiation $(1.5405 \AA$ ) and a graphite monochromator (the step size being of $2 \theta=0.02^{\circ}$ and a 2 seconds time per step) using a grazing incidence configuration (GI-XRD). 


\section{RESULTS}

We present in Table 1 atomic oxygen/bismuth ratios obtained by EDS for SBT films without and with oxygen implantation. These ratios do not provide quantitative information because the oxygen content comes from the SBT film and also from the $\mathrm{SiO}_{2}$ sub-layer of the substrate. Nevertheless, they provide a qualitative indication of the oxygen gain due to the implantation treatment.

TABLE I: Oxygen/bismuth ratio of SBT films.

\begin{tabular}{|l|l|l|}
\hline Sample & Plasma Treatment & Atomic O/Bi ratio \\
\hline S0 & Non treated & 9.1 \\
\hline S1 & One pulse & 9.9 \\
\hline S6 & Six pulses & 10.7 \\
\hline
\end{tabular}
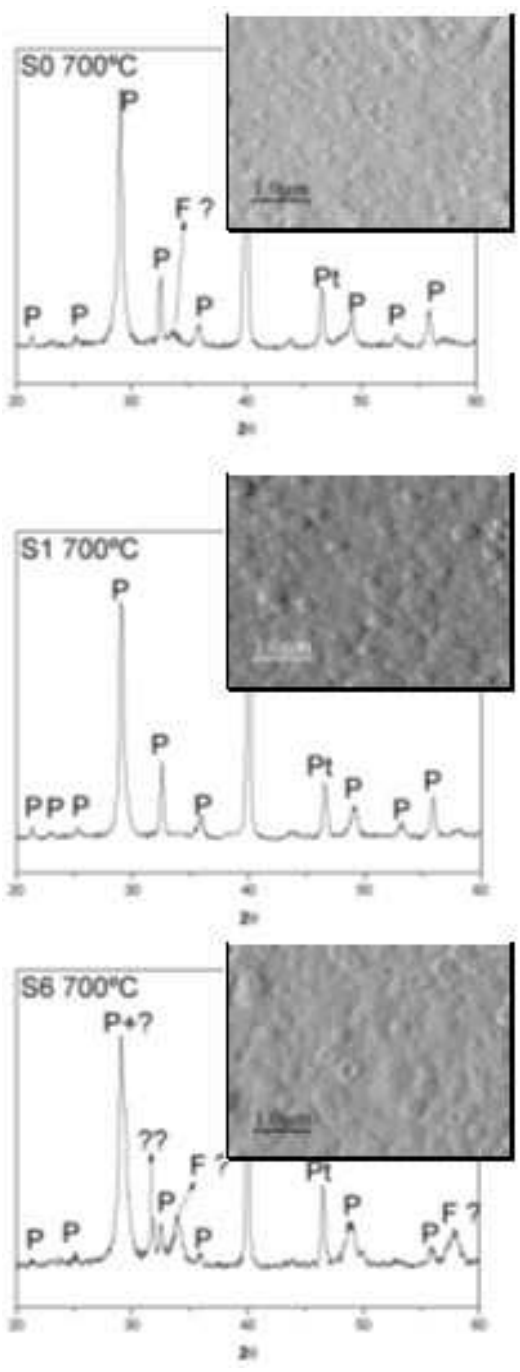

FIG. 5: XRD spectrums and AFM images of samples annealing at $700^{\circ} \mathrm{C}$ (from top to bottom): a) S0; b) S1 c) S6.

AFM images of the as-prepared SBT films (at $520^{\circ} \mathrm{C}$ ) with- out and with oxygen implantation are shown in Figure 2. The non-implanted sample (hereafter called S0) exhibits the characteristic rosettes of the fluorite phase (Fig. 2a). Figures $2 b$ and $2 \mathrm{c}$ show images of the samples implanted with one pulse (S1) and six pulses (S6), respectively. It is evident that the implantation of oxygen amorphize the film surface. This effect is more pronounced for the sample S6. However, the XRD spectra of the tree samples (not shown here) display similar characteristics, exhibiting a broad peak centred at around $2 \theta=29^{\circ}$ which corresponds to the fluorite phase.

Figures $3 \mathrm{a}, 3 \mathrm{~b}$ and $3 \mathrm{c}$ show AFM images and XRD spectra (inserts) for the S0, S1 and S6 samples annealed at $600^{\circ} \mathrm{C}$. Although the XRD spectra do not denote significant differences, the implanted films show a more homogeneous and defined morphology, with larger grain sizes. Samples annealed at $640^{\circ} \mathrm{C}$ (results not shown here) display larger grain sizes in comparison with the ones annealed at $600^{\circ} \mathrm{C}$, but the appearance of the SBT perovskite phase is not detected by XRD.

The XRD spectra of the S0 and S1 samples annealed at $675^{\circ} \mathrm{C}$ reveal characteristic diffraction peaks of the SBT perovskite phase (Figures $4 \mathrm{a}$ and $4 \mathrm{~b}$ ). However, the spectrum of the S0 sample displays less defined perovskite peaks and a more intense peak centred at $2 \theta=33.90^{\circ}$, indicating the presence of a larger content of residual fluorite phase. The surface of the S1 film (insert) exhibits a more defined grain structure in comparison with the SO sample.

Finally, the annealing treatment at $700{ }^{\circ} \mathrm{C}$ developed a well crystallized SBT perovskite structure in the S1 sample, without the presence of spurious phases (Fig. 5b). The S0 sample exhibits less defined perovskite peaks and also shows the presence of peaks which could be ascribed to residual fluorite phase (Fig. 5a). The S6 sample (treated with six pulses during the ion implantation) is degraded; its XRD spectrum exhibits additional peaks that could not be ascribed to any expected phase. These peaks would come from spurious phases formed at the film-substrate interface during the oxygen implantation. This indicates that the experimental set-up energy must be improved in order to control the penetration depth of oxygen ions during the implantation process.

\section{CONCLUSIONS}

The effects of oxygen incorporation, by ion implanting, on the crystallization behaviour of SBT thin films were investigated. The incorporation of oxygen into the intermediate fluorite phase produces a better crystallization of the SBT perovskite phase, increasing the driving force for the fluorite $\rightarrow$ perovskite transformation. These preliminary results indicate that the implantation of oxygen by a Plasma Focus is a promising technique to lower the processing temperature of SBT thin films.

\section{Acknowledgement}

The authors wish to acknowledge the financial support of the Agencia Nacional de Promoción Científica y Tecnológica and Consejo Nacional de Investigaciones Científicas y Técnicas (Argentina). 
Thanks to Dr. M. Ávalos,Ing. Aldo Marenzana, Ing. Guillermo Pochettino, Tec. Horacio Merayo, Tec. Daniel
Castellani, Tec. Raúl Barrionuevo and to the Instituto de Física Rosario.
[1] O. Auciello, J. Scott, and R. Ramesh, Physics Today 51, 22 (1998).

[2] J. F. Scott, Ferroelectric Memories, Springer Series in Advanced Microelectronic 3, Springer-Verlag (2000).

[3] R. W. Schwartz, Chem. Mater. 9, 2325 (1997).

[4] M. G. Stachiotti, R. Machado, A. Frattini, N. Pellegri, and O. de Sanctis, Journal of Sol-Gel Science and Technology, 36, 56 (2005).

[5] M. G. Stachiotti, R. Machado, A. Frattini, N. Pellegri, and O. de Sanctis, Effects of the chelating agent on the fabrication of SBT thin films: Part I. Stoichiometry and crystallization behav- ior, Ferroelectrics, in press (2005).

[6] A. Frattini, R. Machado, M. L. Santiago, N. Pellegri, M. G. Stachiotti, R. Bolmaro, and O. de Sanctis, Effects of the chelating agent on the fabrication of SBT thin films: Part II. Microstructural properties, Ferroelectrics, in press (2005).

[7] G. Sánchez, G. Grigioni and J. Feugeas, Thermal effect of ion implantation with ultra-short ion beams Surface and Coatings Technology, 70 (1995).

[8] G. Sánchez and J. Feugeas, J. Phys. D: Appl. Phys. 30, 927 (1997). 